\title{
Inventario de Estrategias de Control de Recursos: Validación en preadolescentes chilenos
}

\author{
Eduardo Franco Chalco ${ }^{1}$, Christian Berger Silva ${ }^{2}$ \\ Pontificia Universidad Católica de Chile ${ }^{1,2}$
}

\begin{abstract}
La Teoría de Control de Recursos (Hawley, 1999) se ha presentado como una teoría alternativa del estudio de la conducta social en preadolescentes. Sin embargo, carece de instrumentos para evaluar sus propuestas en el idioma espańol. El presente estudio tuvo por objetivo validar el Inventario de Estrategias de Control de Recursos para la población de preadolescentes chilenos. Para alcanzar dicho objetivo se utilizaron metodologías cualitativas y cuantitativas. Se entrevistaron a 7 estudiantes y se encuestaron 180 estudiantes de 5 to y 8 vo básico de dos escuelas públicas de Santiago de Chile. Posterior a la traducción y adaptación, el inventario tuvo un excelente ajuste en el modelo factorial y la validación de criterio obtuvo resultados similares a los reportados en la literatura actual.
\end{abstract}

Palabras clave: Teoría de Control de Recursos, Prosocial, Coerción, Preadolescentes, Estatus.

\section{Resource Control Strategies Inventory: Adaptation in Chilean Preadolescents}

Resource Control Theory (Hawley, 1999) has been presented as an alternative theory for the study of preadolescent social behavior. However, there are no instruments to assess its proposals for the Spanish language. The study's objective was to validate the Resource Control Resources Inventory for the Chilean preadolescent population. For this purpose, qualitative and quantitative methodologies were used. $5^{\text {th }}$ to $8^{\text {th }}$ graders from two public schools from Santiago de Chile were part of the sample. Seven students were interviewed, and 180 were surveyed. After the translation and adaptation, the inventory showed an excellent fit to the factorial model, and the criterion validity showed similar results to those reported in recent literature.

Keywords: Resource Control Theory, Prosocial, Coercion, Preadolescents, Status.

\section{Inventario de Estratégias de Controle de Recursos: Adaptação em Pré-adolescentes Chilenos}

A Teoria do Controle de Recursos (Hawley, 1999) foi presentada como uma teoria alternativa a o estudo da conduta social nos pré-adolescentes. Não obstante, não ha instrumentos pra examinar suas propostas no idioma espanhol. O presente estúdio tinha pelo objetivo

1 Candidato doctoral en Psicología de la Pontificia Universidad Católica de Chile. Interesado en la conducta social y su relación con el estatus durante la adolescencia temprana. Correspondencia a: Av. Vicuña Mackenna 4860, Macul, Santiago, Chile 7820436. E-mail: efranco1@uc.cl

2 Doctor en Psicología Educacional de la Universidad de Illinois. Sus intereses son con las relaciones de pares entre adolescentes y el desarrollo socioemocional durante esta etapa. Correspondencia a: Av. Vicuńa Mackenna 4860, Macul, Santiago, Chile 7820436. E-mail: cberger@uc.cl 
validar o Inventario de Estratégias de Controle de Recursos pra uma população de pré-adolescentes chilenos. Pra alcançar o objetivo metodologias quantitativas e qualitativas foram utilizadas. Sete estudantes foram entrevistados e 180 estudantes foram pesquisados de 5 to a 8vo básico de duas escolas publicas de Santiago de Chile. Em seguida a tradução e adaptação, o inventario teve um excelente ajuste no modelo fatorial, e a validação de critério conseguiu resultados similares a os reportados na literatura atual.

Palavras-chave: Teoria de Controle de Recursos, Prosocial, Coação, Pré-adolescentes, Status.

Inventaire des Stratégies de Contrôle des Ressources: Adaptation en Préadolescentes Chiliens

La Théorie du Contrôle des Ressources (Hawley, 1999) a été présentée comme une théorie alternative à l'étude du comportement social chez les préadolescents. Cependant, il n'existe aucun instrument pour examiner vos propositions en espagnol. Cette étude visait à valider l'Inventaire des Stratégies de Contrôle des Ressources pour une population de préadolescents chiliens. Pour atteindre cet objectif, des méthodologies quantitatives et qualitatives ont été utilisées. Sept élèves ont été interrogés et 180 élèves ont été questionné de la cinquième à la huitième année d'éducation de base dans deux écoles publiques à Santiago du Chili. Après traduction et adaptation, l'inventaire s'intégrait parfaitement dans le modèle factoriel et la validation des critères donnait des résultats similaires à ceux rapportés dans la littérature actuelle.

Mots-clés: Théorie du Contrôle des Ressources, Prosocial, Contrainte, Préadolescents, Statut. 
La conducta agresiva ha sido concebida generalmente como una respuesta proveniente del mal procesamiento de información sobre el contexto social (Crick \& Dodge, 1994). Sin embargo, perspectivas más recientes proponen que la agresión es en realidad una respuesta adaptativa a las demandas del contexto (Hawley, Little et al., 2007), que, entre alguna de sus funciones, sirve como instrumento para la obtención de estatus entre sus pares (Berger \& Rodkin, 2012).

Una perspectiva alineada con esta visión es la Teoría de Control de Recursos (TCR) propuesta por Patricia Hawley (1999). Esta teoría tiene una perspectiva evolucionista, plantea que los seres humanos tienen disposiciones que les impulsan a buscar dominancia social, debido a que los sujetos con estatus prominentes tienen mayor acceso a recursos que facilitan su supervivencia y reproducción. Para Hawley (2003a) la dominancia social se define como el máximo control de recursos materiales y sociales. De acuerdo con esta teoría, el concepto de recurso hace referencia a una amplia variedad de objetos y situaciones. Por ejemplo, los recursos materiales se refieren a alimentos, dinero, etc.; y los recursos sociales hacen referencia a amistades, estatus, influencia, etc. Por tanto, a diferencia de la concepción etológica de la dominancia social, la propuesta de la TCR es que aquel individuo que sea capaz de utilizar diversas estrategias para controlar la mayor cantidad de recursos será el más dominante. En este sentido Hawley (2003a) propone que desde tempranas etapas del desarrollo se van poniendo en práctica múltiples estrategias para obtener dominancia social, pudiendo ser identificadas dos formas principales: estrategias prosociales y estrategias coercitivas.

Las estrategias prosociales son comprendidas como conductas socialmente aceptadas para obtener recursos en interacción con otras personas; en este sentido, se pueden enumerar conductas como prometer reciprocidad, cooperación, o formación de alianzas. En ese sentido las estrategias prosociales se pueden entender como un mecanismo de 
negociación con otros para obtener algo a cambio. Por otro lado, las estrategias coercitivas implican conductas como la intimidación, manipulación, agresión, etc. realizadas de manera intencional para obtener recursos (Hawley, 2007). Por tanto, se puede decir que las estrategias coercitivas son utilizadas para forzar a otros a cumplir la voluntad del individuo que las realiza.

A pesar de que ambas formas de estrategias de control de recursos parecen ser opuestas, es necesario tomar en cuenta que ambas comparten un mismo fin, la obtención de recursos. Esto se relaciona con un aspecto nuclear de la TCR, la hipótesis de centralidad social. Esta propone que los individuos que alcanzan el más alto nivel de dominancia social son aquellos que son capaces de combinar ambos tipos de estrategias en diversas situaciones (Hawley, 1999). A estos individuos se les ha llamado controladores biestratégicos y son quienes en comparación con otros individuos de su grupo social utilizan con más frecuencia ambas estrategias, logrando así una mayor obtención de recursos a través del uso de conductas acorde con la situación social en que se encuentren. Es importante destacar que el uso de las estrategias de control de recursos es intencional y está orientado a cumplir objetivos sociales. Es por este motivo que a los controladores biestratégicos también se les ha llamado "Maquiavélicos", dado que están dispuestos a usar las estrategias que sean necesarias para alcanzar sus metas (Hawley, 2003b).

Las estrategias de control de recursos no deben ser confundidas con la conducta agresiva y conducta prosocial en general. La agresión y la prosocialidad pueden también tener formas reactivas (Findley-van Nostrand \& Ojanen, 2018; Prinstein \& Cillessen, 2003), en cambio las estrategias de control de recursos son siempre instrumentales o proactivas (Hawley, 1999). Esto puede observarse en los consistentes resultados de investigaciones que encuentran una correlación positiva entre las estrategias coercitivas y prosociales (aprox. $r=.40$, Hawley, 2007) y la correlación negativa o nula entre la conducta agresiva y prosocial (Berger et al., 2015; Cillessenet al., 2013).

A pesar de lo mencionado anteriormente, las estrategias de control de recursos se encuentran relacionadas con estas formas de conducta 
social. Específicamente se observan relaciones entre las estrategias coercitivas con la conducta agresiva (agresión relacional: $r=.63$; agresión física: $r=.61$, Findley \& Ojanen, 2013) y las estrategias prosociales están relacionadas a la conducta prosocial ( $r=.14$, Findley \& Ojanen, 2013), aunque esta última tiene una magnitud inferior, indicando que son constructos similares, pero mantienen algunas diferencias.

\section{Enfoque centrado en la persona}

Un aspecto central que diferencia a la TCR de otras teorías en la literatura de relaciones de pares es enfoque centrado en la persona. De este modo, se identifican subgrupos de controladores de recursos presentes en todos los contextos. Para tal objetivo Hawley (2003b) propuso la identificación de 5 grupos a partir de la frecuencia del uso de cierta forma de estrategia de control de recursos, con cortes arbitrarios en los percentiles de cada una de las dimensiones de control coercitivo y prosocial, es a partir de estos cortes que se identifican los controladores biestratégicos (superior al percentil 66 en ambas estrategias). También se identifican controladores prosociales (superior al percentil 66 en estrategias prosociales, e inferior al percentil 66 en estrategias coercitivas), controladores coercitivos (superior al percentil 66 en estrategias coercitivas, e inferior al percentil 66 en estrategias prosociales), controladores típicos (uso promedio de ambas estrategias) y no controladores (inferior al percentil 33 en ambas estrategias).

Con este enfoque ha sido posible corroborar algunos aspectos de la hipótesis de centralidad social. Por ejemplo, se ha reportado que los controladores biestratégicos tienden a ser los más populares dentro de su grupo de pares, mientras que los no controladores tienden a ser los menos populares. Con respecto a los controladores prosociales, se ha encontrado que suelen ser los estudiantes con mayor aceptación social, sin embargo, no suelen tener el mismo nivel de popularidad que los controladores biestratégicos (Hawley, 2003b). Del mismo modo, también se ha identificado que los controladores biestratégicos utilizan 
la agresión y el bullying como instrumentos para obtener dominancia en su grupo de pares (Olthof et al., 2011). Mientras que, los no controladores tienden a mayor retraimiento social que el resto de sus compañeros (Findley \& Ojanen, 2013).

Con respecto a las diferencias de género, inicialmente se propuso que, dado que las estrategias de control de recursos son variadas y no solamente implican el uso de la fuerza física para lograr coerción, no existirían diferencias de género (Hawley, 1999). Sin embargo, la evidencia ha demostrado que tanto la razón de uso de las distintas estrategias como los porcentajes de clasificación en cada uno de los grupos de control son distintos entre hombres y mujeres. Las mujeres en general tienden a preferir el control prosocial mientras que los hombres reportan mayor uso de estrategias de control coercitivo (Findley \& Ojanen, 2013; Hawley, 2003b; Reijntjes et al., 2017). Por tanto, también se pueden identificar diferencias a nivel de clasificación en los grupos de control de recursos, donde para el grupo de control hay una mayor representación de hombres, mientras que para el grupo de control prosocial se observan en mayor medida mujeres; sin embargo, no se encuentan diferencias de representación en los otros grupos de control (Reijntjes et al., 2017).

\section{Medición de las estrategias de control de recursos}

Desde la postulación de la TCR, las estrategias de control de recursos han tenido múltiples modos de ser medidas. A través de nominaciones de pares (Findley \& Ojanen, 2013; Olthof et al., 2011), reporte de profesores (Hawley, Johnson et al., 2007), autoreporte (Hawley, 2003b; Olthof et al., 2011) y observación (Hawley, 2003a). Casi todas estas mediciones han tenido como base los ítems de ejemplo propuestos por Hawley (2003b), que posteriormente se publicaron en un instrumento de autoreporte completo para adultos, denominado Inventario de Estrategias de Control de Recursos (IECR, Hawley, 2006). Este instrumento solo se encuentra disponible en el idioma inglés. 
De acuerdo con la revisión de literatura, solamente existe un instrumento para preadolescentes y adolescentes, propuesto por Olthof et al. (2011). Este instrumento tiene algunas diferencias con el IECR en la redacción de los ítems. Específicamente, estos autores mencionan que el IECR utiliza frases como: "Influencio a otros haciendo algo por ellos a cambio". Que implica que las estrategias utilizadas ya alcanzan un objetivo, y no solamente harían referencia a las conductas que despliegan para alcanzar dicho objetivo independientemente de si lo logran o no. Un contra argumento a esta propuesta es que el control logrado es justamente el objetivo de la teoría, básicamente se quiere conocer como es que los adolescentes logran influenciar en su entorno. Por tanto, el logro del objetivo social propuesto es indispensable para conocer si la estrategia utilizada es beneficiosa para la persona que la despliega.

Por otro lado, la cultura Latinoamericana ha sido descrita como colectivista por encima de individualista (Fuligni et al., 1999), por lo que las estrategias de control descritas para poblaciones europeas y estadounidenses podrían tener estructuras distintas. Específicamente, se esperaría que las estrategias relacionadas a la manipulación de amistades para el beneficio propio no serían socialmente aceptadas, por lo que habría mayor deseabilidad social con este tipo de respuestas. De este modo se resalta la importancia del estudio de la TCR en distintas culturas para conocer probables diferencias en la estructura factorial del IECR dependiendo de los valores de las distintas sociedades, y así atenuar posibles sesgos culturales de la investigación.

En resumen, desde un punto de vista psicométrico es necesario identificar contextualmente cuales son las estrategias de control de recursos utilizadas por los preadolescentes chilenos, y evaluar si estas pueden ser diferenciadas claramente como prosociales o coercitivas. Del mismo modo identificar si son equivalentes entre hombres y mujeres, dadas las diferencias de género encontradas en la literatura. Por los motivos expuestos anteriormente, el objetivo principal del presente estudio fue validar el Inventario de Estrategias de Control de Recursos (IECR). Para lograr dicho objetivo se utilizaron metodologías cuantitativas y cualitativas, que siguieron los siguientes pasos: 
traducción y retrotraducción, adaptación de los ítems para una muestra adolescente, juicio de expertos, evaluación psicométrica y entrevista grupal. Del mismo modo, para la validación se espera replicar los resultados de la literatura expuesta en cuanto a la formación de grupos de estrategias de control de recursos y sus subsecuentes niveles de popularidad.

\section{Método}

\section{Participantes}

La recolección de datos del presente estudio tuvo dos momentos, ambos fueron realizados durante la primavera del 2017. Para esto, en un primer momento se encuestaron a 180 estudiantes de $5^{\circ}$ a $8^{\circ}$ ańo de enseńanza general básica de dos escuelas de la Región Metropolitana de Santiago, Chile. Mientras que en un segundo momento se entrevistó grupalmente a 7 estudiantes de una de las escuelas anteriormente encuestadas. La edad promedio fue de 12 años $(D E=1.27, \mathrm{Min}=10$, Max $=15)$. La muestra estuvo balanceada por sexo ( $52 \%$ mujeres). Con respecto al nivel socioeconómico de las escuelas encuestadas, la Agencia de Calidad de la Educación Chilena (SIMCE) clasifica a una de las escuelas como medio bajo y a la otra escuela como medio alto.

\section{Medición}

Inventario de Estrategias de Control de Recursos (IECR, Hawley, 2006). Este cuestionario de autoreporte consta de 12 ítems medidos en una escala de Likert de 4 puntos ( 1 = Nunca, 2 = Pocas veces, $3=$ Muchas veces, 4 = Siempre). El instrumento mide las dos formas de estrategias de control de recursos (prosocial y coercitiva). Este instrumento fue originalmente diseñado para población adulta y angloparlante, por lo que se siguieron varias etapas para su traducción y adaptación.

Inicialmente se realizó la traducción y retro traducción del instrumento (Brislin, 1970). En un primer momento, dos psicólogos 
bilingües realizaron una traducción individual, y por separado, del instrumento para posteriormente integrar las dos versiones en una sola llegando a consenso en donde hubiera alguna diferencia. A continuación, un tercer psicólogo bilingüe, que no participó de la traducción inicial, y que no tenía conocimiento del contenido original de los ítems en inglés realizó la retro traducción del español al inglés. Esta versión fue comparada con la original donde no se observaron discrepancias mayores, por lo cual se procedió a adaptar los ítems para la edad y contexto de la muestra. Los ítems fueron redactados de modo que puedan ser comprendidos por escolares de 9 a 15 años y que además mantengan la validez de constructo. Posteriormente se procedió a la fase de juicio de expertos, por lo que la versión resultante del instrumento de la fase anterior fue enviada a 5 expertos en el área para que respondan dos preguntas respecto de cada ítem: "¿el presente item mide una estrategia coercitivalprosocial de control de recursos?" y "jel sentido de este item puede ser entendido por un escolar en Chile de entre 9 y 15 años?". Del mismo modo, se permitió que los expertos hicieran comentarios respecto a la adecuación de los ítems para la muestra y constructo. La información recolectada durante esta fase fue utilizada preparar una tercera versión del instrumento cuyos ítems, así como a la dimensión a la que pertenecen, se encuentran en la Tabla 1.

Popularidad. Los estudiantes reportaron cuán populares se percibían a sí mismos a través de la siguiente pregunta: "En una escala del 1 al 10, y en comparación con tus compañeros ¿cuán popular eres?”

Entrevista. Se diseñó un guion de entrevista grupal donde se leyó cada uno de los ítems del IERC y posteriormente se les preguntó a los participantes “Qué entienden ustedes al leer esta afirmación?” y "Piensan ustedes que hacer esto es algo común entre personas de su edad?" Estas preguntas fueron abiertas a todos los participantes y se incentivó a que todos dieran al menos una respuesta para conocer las impresiones tanto generales como específicas respecto de cada ítem del instrumento. Del mismo modo se permitió que los participantes hicieran comentarios con respecto de la temática tratada, para poder determinar la comprensión general del constructo. 


\section{Procedimiento}

Inicialmente se contactó a los directores de las escuelas seleccionadas, estos brindaron su autorización después de que se les explicara el proyecto de investigación. En un segundo momento se enviaron cartas de consentimiento informado a los apoderados donde escogían dar autorización a la participación de sus hijos en la aplicación de las encuestas y/o en la entrevista grupal, solamente aquellos estudiantes autorizados participaron del estudio. En un tercer momento dos investigadores ingresaron a las aulas en el horario regular de clases para realizar la aplicación de los cuestionarios, previa coordinación con los profesores encargados. Se solicitó que los estudiantes que no fueron autorizados a participar trabajaran en otras actividades escolares mientras sus compañeros realizaban la encuesta. A los estudiantes con consentimiento se les leyó una carta de asentimiento informado, expresándoles la voluntariedad de su participación y solo aquellos que firmaran el asentimiento formarían parte de la muestra final del estudio. Aquellos estudiantes que se rehusaron a participar, se les solicitó trabajar en otras actividades escolares. La aplicación de los instrumentos demoró aproximadamente 30 minutos. Ambos investigadores estuvieron durante toda la aplicación para responder preguntas y asegurar la confidencialidad de las respuestas. Una vez finalizada la aplicación de los instrumentos se agradeció a los participantes y continuaron las actividades regulares de las clases.

Posteriormente, una de las escuelas fue seleccionada al azar para realizar la entrevista grupal. Se seleccionaron 2 estudiantes aleatoriamente de cada uno de los niveles educativos de la muestra, siendo un total de 8 participantes. A continuación, se solicitó a la escuela un espacio privado donde se pueda realizar la entrevista en el horario de clases. $\mathrm{Al}$ momento de iniciar la entrevista estuvieron presentes tres investigadores, uno que era encargado de dirigir la entrevista de acuerdo con el guion y otras dos investigadoras como colaboradoras. Se leyó una carta de asentimiento informado recalcando la voluntariedad de esta instancia. Un estudiante decidió voluntariamente abstenerse de participar por lo que regresó a su salón. A los participantes restantes se les 
acentuó la importancia de mantener la confidencialidad y el respeto a sus compañeros con sus respuestas. Una vez terminada la entrevista se agradeció a los participantes y fueron enviados nuevamente a sus salones respectivos. La entrevista fue transcrita por una persona calificada la cual firmó un compromiso de confidencialidad. Todos los procedimientos de este estudio fueron aprobados por el Comité de Ética de la universidad patrocinante.

\section{Análisis de datos}

El análisis de datos tuvo dos momentos, uno para los datos cualitativos y otro para los datos cuantitativos. La transcripción de la entrevista grupal fue analizada mediante categorías previamente definidas con la información de la revisión bibliográfica. Se buscó que cada uno de los ítems coincidiera con dichas categorías para considerar que fueron entendidos correctamente. Las conclusiones obtenidas durante este análisis sirvieron para alimentar y guiar el análisis cuantitativo. Inicialmente se hicieron análisis descriptivos y correlacionales que fueron interpretados a la luz de los datos cualitativos. Para ilustrar esto se integran vińetas de la entrevista para apoyar el análisis (los nombres de los participantes son ficticios). En este punto se decidió retirar tres ítems que no fueron correctamente comprendidos y se propuso una estructura factorial que posteriormente fue probada en un Análisis Factorial Confirmatorio a través del estimador de Mínimos Cuadrados Ponderados con Medias y Varianzas Ajustadas (WLSMV, por sus siglas en inglés) debido a la naturaleza ordinal de 4 categorías de los ítems. Los criterios para determinar el ajuste del modelo fueron en términos absolutos que el Chi-cuadrado no sea significativo $(p>.05)$, y que en términos relativos el TLI y CFI sean mayores a .95 , además que el RMSEA y $S R M R$ sean menores a .08 (Hooper et al., 2008). Como siguiente paso se contrastaron los distintos niveles de invarianza métrica del modelo por género. Finalmente, para explorar la validez de criterio se clasificó a los estudiantes en los cinco tipos de estrategias de control de recursos de acuerdo con la propuesta de Hawley (2003b) y se determinaron las diferencias a nivel de popularidad a través de un Análisis de Varianza con contrastes Post-Hoc con 
ajuste de Bonferroni. Todos los análisis fueron realizados utilizando $\mathrm{R}$ v3.5.1 (R Core Team, 2018), y para los modelos de variables latentes se utilizó el paquete "lavaan" (Rosseel, 2012).

\section{Resultados}

\section{Análisis descriptivo}

Comenzando con los ítems de la dimensión de control prosocial se puede observar en la Tabla 1 que el ítem 9 posee la media y desviación estándar más baja de la dimensión $(M=1.23 ; D E=.58)$, indicando que la mayoría de los estudiantes de la muestra indicó que no forma nuevas amistades para conseguir que los otros cumplan su voluntad. El análisis de lo informado por los adolescentes en la entrevista grupal da cuenta que este ítem no fue comprendido adecuadamente, puesto que las respuestas de los participantes hacían referencia a amistades actuales, en vez de formar nuevas amistades de manera instrumental para obtener algo a cambio.

"[...] Yo soy amigo del Fernando, de verdad, somos amigos y yo le digo [...] que me dé algo o me compre algo, diciéndole que yo soy su amigo." (Manuel, 11 años)

Además, se pude observar en la Tabla 2 que este ítem está positivamente correlacionado con el ítem 5 el cuál hace referencia a una estrategia similar $(r=.22 ; p<.01)$, donde se forma una amistad, pero sin un componente afectivo por lo cual sería una estrategia coercitiva.

En cambio, el resto de los ítems de la escala de estrategias prosociales muestran tener medias y varianzas ligeramente más altas, y se encuentran de leve a moderadamente relacionados entre si $(.10>r s<.53)$. Esto indicaría al menos un nivel mínimo de consistencia entre los ítems utilizados para dicha escala. La escala de estrategias prosociales sin el ítem 9 alcanza una aceptable consistencia interna medida a través del Alfa de Cronbach (.70). 


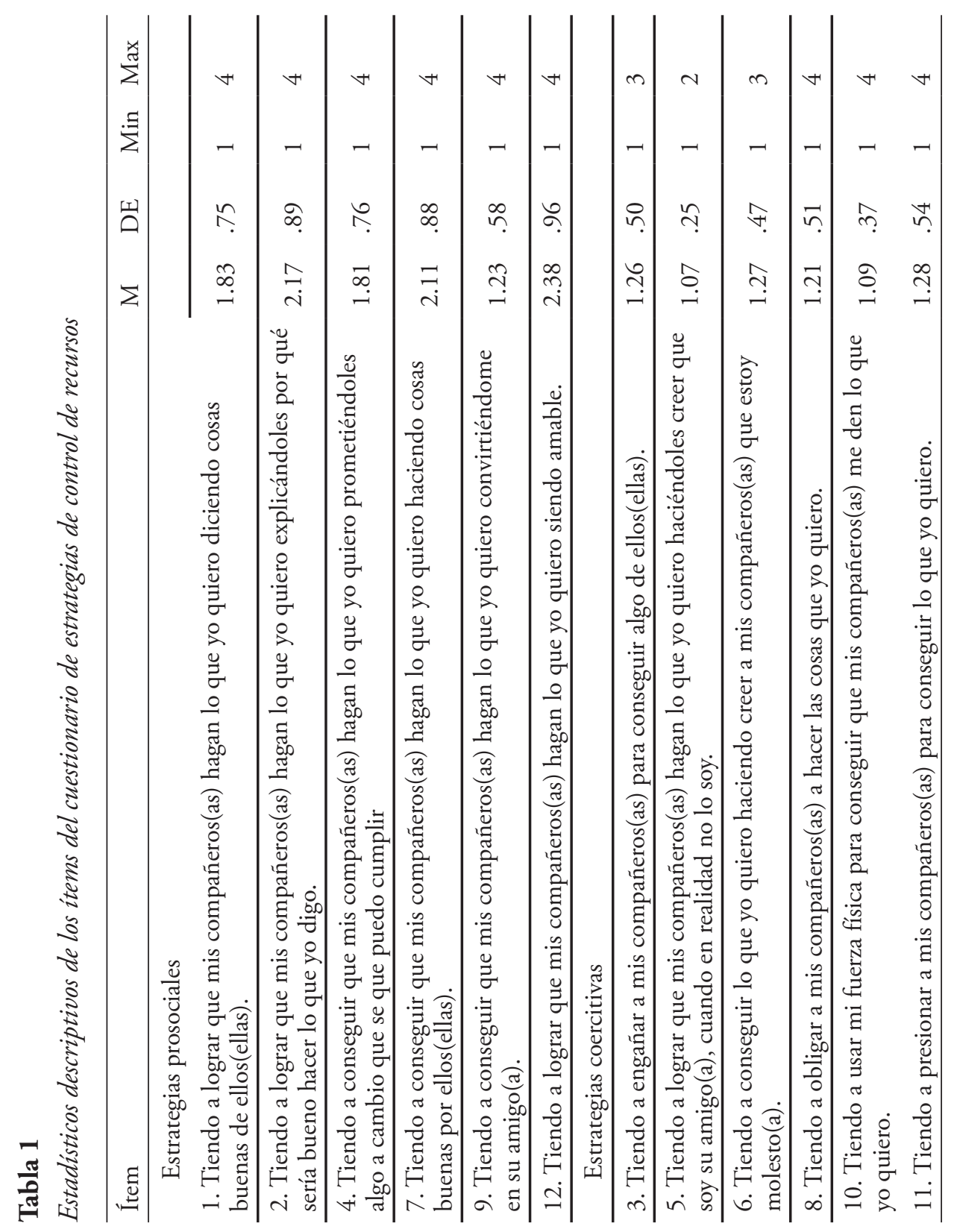


Con respecto a la escala de estrategias coercitivas, el ítem 5 tiene la media y desviación estándar más baja de todo el instrumento $(M=$ 1.07; $D E=.25$ ), siendo esta la estrategia menos reportada. Al observar las respuestas de la entrevista grupal, este el ítem 5 es difícil de entender y además se observa una valoración negativa del ítem, indicando que podría ser altamente susceptible a respuestas por deseabilidad social:

"Tú les dices que somos amigos, pero en realidad no lo son, como que tú te estás engańando a ti mismo y quizá te puedes estar haciendo a ti mismo un mal por no ser amigos y engañarlo a él y también a ti” (Manuel, 11 ańos)

Del mismo modo, el ítem 10 presenta un promedio bajo $(M$ $=1.09 ; D E=.37)$. La entrevista grupal da cuenta que el ítem es correctamente comprendido por los participantes, puesto que el uso de fuerza física es una estrategia que pudieron observar en otros compańeros. Sin embargo, esta estrategia también fue identificada como una conducta que merece castigo, indicando la susceptibilidad de este ítem a respuestas por deseabilidad social:

"En mi antiguo colegio $[\ldots]$ un nińo de sexto estaba obligando a uno de quinto a que le comprara algo y le pegó. [...] Eeh... luego llegó la directora y le dijo que no lo tenía que hacer, pero él nunca cambió. Siempre le pegaba a los demás para que hagan las cosas que quería." (Francisco, 10 años).

Otros dos ítems con promedios bajos en esta escala fueron los ítems $3(M=1.26 ; D E=.50)$ y $6(M=1.27 ; D E=.47$; respectivamente). En la entrevista grupal se pudo observar que los participantes pudieron comprender el sentido de las preguntas, pero ambas tuvieron una valencia negativa. Sin embargo, estos ítems se encuentran consistentemente relacionados con los otros ítems de la escala sin considerar los ítems 5 y $10(.17>r s<.35)$. La escala de estrategias coercitivas sin los ítems 5 y 10 alcanza una consistencia interna aceptable a través del Alfa de Cronbach (.60). 


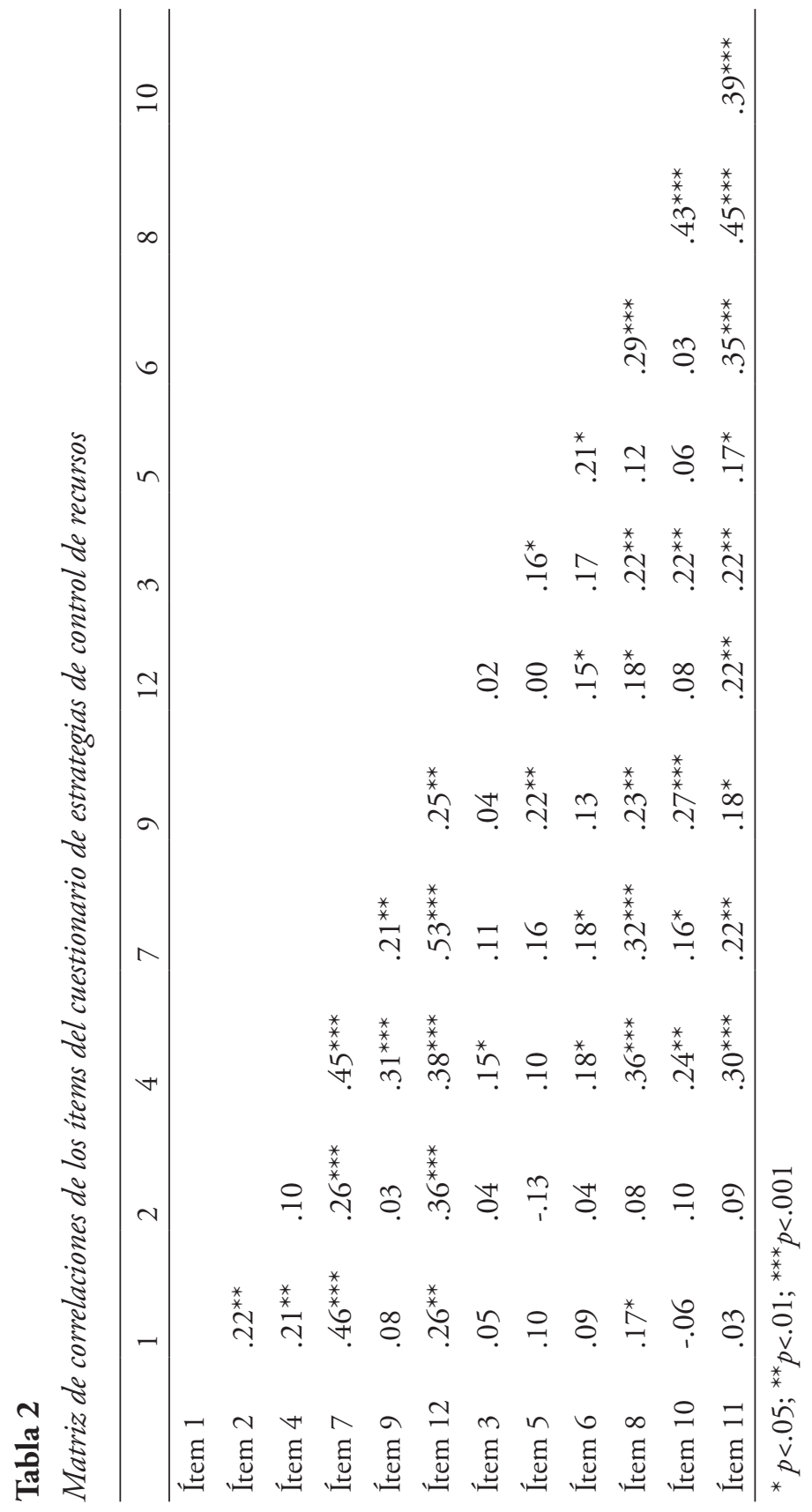




\section{Modelo de Análisis Factorial Confirmatorio}

Dados los resultados previos se propuso un modelo estructural con dos dimensiones. La dimensión de estrategias prosociales fue medida por los ítems 1, 2, 4, 7, y 12. Mientras que, la dimensión de estrategias coercitivas fue medida por los ítems 3, 6, 8 y 11; excluyendo de este modo los ítems 5, 9 y 10. Con respecto al ajuste general del modelo, se obtuvo un buen ajuste en términos absolutos y relativos $\left(X^{2}=17.94 ; g l=\right.$ 26; $p=.88 ; C F I=1 ; T L I=1.04 ; R M S E A=0 ; S R M R=.05)$. El resultado del análisis se puede ver diagramado en la Figura 1. Se puede observar que en ambas dimensiones todas las cargas factoriales son significativas y positivas. Además, se observa una correlación moderada y positiva entre las dimensiones de estrategias prosociales y coercitivas $(r=.47 ; p<$ $.001)$.

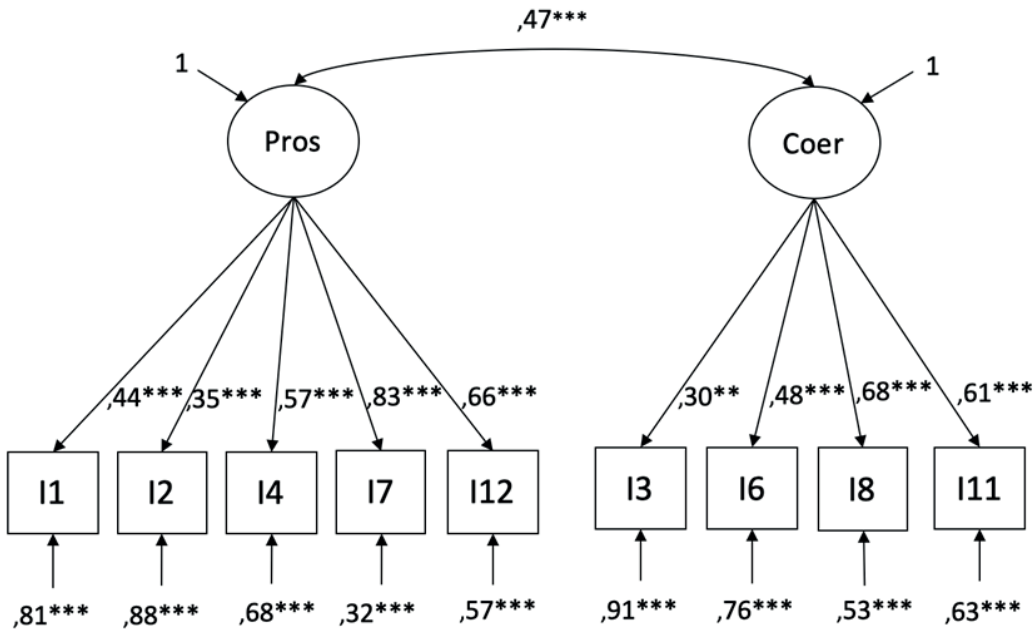

Figura 1. Estructura factorial del Inventario de Estrategias de Control de Recursos

Nota: Pros $=$ Estrategias prosociales. Coer $=$ Estrategias coercitivas. ${ }^{*} p<.05 ;{ }^{* *} p<.01$; $* * * p<.001$ 
En la Tabla 3 se puede observar la prueba de invarianza métrica del inventario entre hombres y mujeres. Los resultados indican que no hay diferencias significativas en la medición de este instrumento entre hombres y mujeres, alcanzando un nivel de invarianza estricta. Esto se puede interpretar como que los constructos de estrategias coercitivas y estrategias prosociales son los mismos para ambos sexos, pudiendo de este modo compararse sus puntajes.

\section{Tabla 3}

Prueba de invarianza métrica entre hombres y mujeres para el Inventario de Estrategias de Control de Recursos

\begin{tabular}{lccccc}
\hline & $g l$ & $X^{2}$ & Delta $X^{2}$ & Delta $g l$ & $p$ \\
\hline Configural & 52 & 36.55 & & & \\
Débil & 59 & 52.56 & 8.61 & 7 & .28 \\
Fuerte & 66 & 59.55 & 7.58 & 7 & .37 \\
Estricta & 68 & 63.03 & 1.13 & 2 & .56 \\
\hline
\end{tabular}

\section{Clasificación y diferencias de género en los grupos de estrategias de control de recursos}

En la tabla 4 se puede observar la clasificación de individuos en cada uno de los grupos de control de recursos. Es posible observar que los grupos con mayor representación fueron los de control prosocial (25.6\%) y los de no control (25.6\%), y el grupo con menor clasificación de estudiantes fue el de control promedio (12.78\%). Se observa en el caso de las mujeres que el grupo más grande es el de no control (26.88\%), mientras que el más pequeño es el de control promedio (15.05\%). Para el caso de los hombres el grupo más numeroso por el contrario fue el de control prosocial (28.74\%), y los grupos más pequeńos fueron los control coercitivo y control promedio $(10.35 \%$ en ambos casos). No se encontraron diferencias significativas en la clasifi- 
cación de los grupos de estrategias de control de recursos entre hombres y mujeres $\left(X^{2}=6.27, g l=4, p=.18\right)$.

\section{Tabla 4}

Clasificación y comparación en los grupos de estrategias de control de recursos diferenciada por sexo

\begin{tabular}{lcccccc}
\hline & \multicolumn{2}{c}{ Mujeres } & \multicolumn{2}{c}{ Hombres } & \multicolumn{2}{c}{ Total } \\
\cline { 2 - 7 } & $N$ & $\%$ & $N$ & $\%$ & $N$ & $\%$ \\
\hline Control Biestratégico & 15 & 16.13 & 23 & 26.44 & 38 & 21.11 \\
Control Prosocial & 21 & 22.58 & 25 & 28.74 & 46 & 25.56 \\
Control Coercitivo & 18 & 19.35 & 9 & 10.35 & 27 & 15.00 \\
Control Promedio & 14 & 15.05 & 9 & 10.35 & 23 & 12.78 \\
No control & 25 & 26.88 & 21 & 24.14 & 46 & 25.56 \\
\hline
\end{tabular}

\section{Validez de criterio}

La figura 2 muestra los promedios de popularidad de cada uno de los grupos de control de recursos. Se realizó un Análisis de Varianza que evidenció diferencias entre al menos uno de dichos grupos $\left(F_{(4,147)}=\right.$ 3.19; $p=.015)$. El análisis post hoc permitió identificar diferencias específicas entre el grupo de controladores biestratégicos y el grupo de control promedio $(p=.023)$. No se lograron identificar más diferencias significativas entre los otros grupos de estrategias de control de recursos $(p>.05)$. 


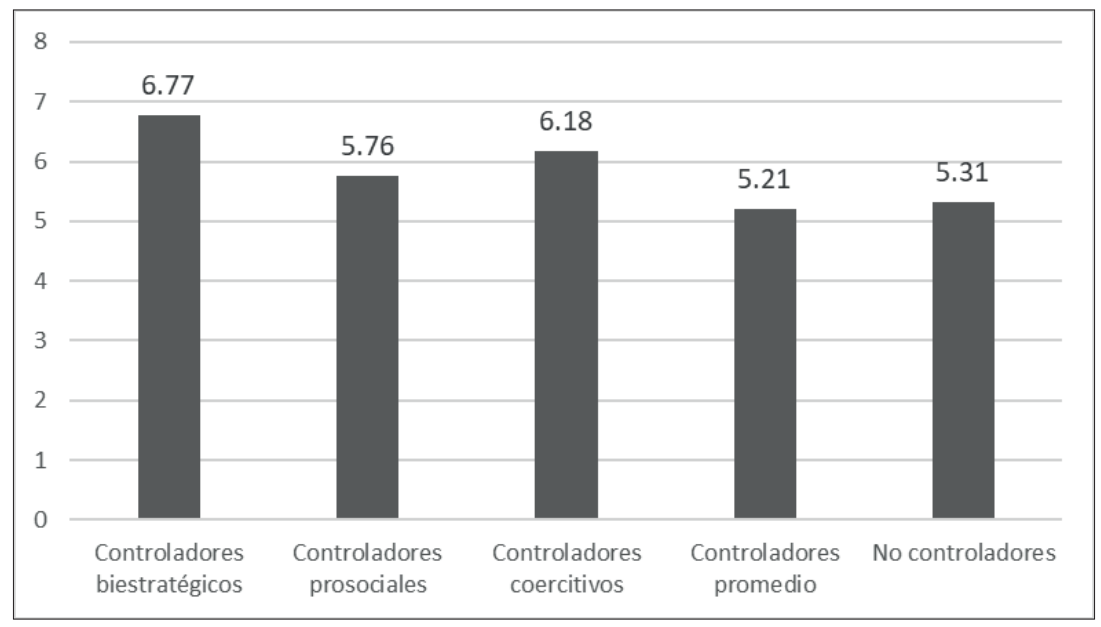

Figura 2. Promedios de popularidad de los grupos de control de recursos

\section{Discusión}

El presente estudio tuvo por objetivo principal traducir, adaptar y validar el IECR para el contexto chileno. Después de la propuesta de una nueva estructura factorial sin los ítems 5, 9 y 10 se pudo observar una aceptable consistencia interna del instrumento, además de un ajuste óptimo en el modelo Factorial Confirmatorio. En este sentido, se puede concluir que las dos dimensiones de las estrategias de control de recursos del IECR están bien definidas y comprendidas por una población de preadolescentes chilenos. La eliminación de los ítems 5, 9 y 10 se fundamentó en el análisis estadístico y en los resultados de las entrevistas, en las cuales se evidenció una alta deseabilidad social y baja comprensión de la estrategia.

La cultura latinoamericana a diferencia de la cultura anglosajona y europea tiene un enfoque más colectivista por sobre lo individual (Fuligni et al., 1999), por lo que es posible que estrategias donde se fabrique ficticiamente una amistad o se use la fuerza física como 
mecanismo para obtener algo a cambio de otra persona pueden tener una carga valórica más negativa que otras estrategias de control de recursos. En este sentido, a pesar de que es posible que los estudiantes en la realidad puedan estar utilizando este tipo de estrategias, estos serán renuentes a reportar su uso. Esto podría ser confirmado con estudios observacionales de las interacciones diarias de los preadolescentes.

En cuanto a las diferencias según sexo para el instrumento, el análisis de invarianza permitió determinar que ambas dimensiones son comprendidas del mismo modo por hombres y mujeres, por lo que sus puntajes son comparables. Sin embargo, es necesario notar que estos resultados no coinciden con lo reportado en la literatura, generalmente se identifica mayor representación femenina en el grupo de estrategias prosociales, y mayor representación masculina en el grupo de estrategias coercitivas de control (Findley \& Ojanen, 2013; Hawley, 2003b; Olthof et al. 2011; Reijntjes et al. 2017). Esto indicaría que la muestra chilena, a diferencia de las europeas y estadounidenses, no tienen una preferencia definida por sexo al momento de instrumentalizar conductas para obtener algo a cambio entre sus compańeros. Otro aspecto importante a resaltar es que el ítem 10 al ser eliminado, las estrategias coercitivas medidas en este estudio no toman en cuenta a la fuerza física como un instrumento para la obtención de recursos, lo cuál también explicaría las diferencias de sexo encontradas con la literatura actual. Es importante resaltar nuevamente la naturaleza intencional e instrumental de las estrategias de control de recursos, ya que en estudios anteriores si se han encontrado diferencias de sexo en el uso de conductas agresivas y prosociales para poblaciones chilenas (Berger et al., 2015).

Los porcentajes en la clasificación de los grupos de estrategias de control de recursos en general son bastante similares a los reportados en otros estudios con preadolescentes. Sin embargo, se identifican algunas diferencias específicas. Los grupos de control prosocial y no control fueron los más numerosos en el presente estudio, con $26 \%$ en ambos casos, mientras que en estudios previos el grupo con mayor representación es el de control promedio (Hawley, 2003b, Olthof et al. 
2011, Reijntjes et al. 2017), y para el presente estudio dicho grupo es el que menor representación tuvo (13\%). Esto indicaría que para la población chilena el uso de estrategias prosociales es más prevalente que en otras poblaciones. Esta diferencia puede deberse a lo mencionado anteriormente respecto de culturas latinoamericanas, las cuales al ser de naturaleza colectivista (Fuligni et al., 1999) aceptarían en mayor medida las estrategias prosociales. Es llamativo, sin embargo, que el mismo nivel de representación se haya podido identificar en el grupo de no control. Esto indica que hay un gran número de preadolescentes chilenos que reporta no utilizar ninguna conducta o estrategia para obtener algo a cambio entre sus pares, siendo este un aspecto que necesitaría ser profundizado en futuros estudios para determinar si es debido a la deseabilidad social o si hay algún factor cultural que haga tan prevalente a este grupo.

Finalmente, y con respecto a la validez de criterio se esperaban encontrar diferencias en los niveles de popularidad reportados por los estudiantes en cada uno de los grupos de estrategias de control de recursos. Los resultados permitieron corroborar parcialmente la hipótesis de centralidad social (Hawley, 1999). Ya que los participantes clasificados como controladores biestratégicos obtuvieron un promedio más alto en popularidad que los estudiantes clasificados como contoladores promedio. Sin embargo, no se encontraron diferencias con los grupos de control prosocial, coercitivo, ni no control. Estos resultados, indican que el uso de ambas estrategias coercitivas y prosociales permiten a los participantes obtener un nivel mayor de popularidad que quienes las utilizan en menor frecuencia. Sin embargo, la ausencia de diferencias entre los grupos de control prosocial, coercitivo y no control con el grupo de biestratégicos no permite dar una conclusión definitiva.

\section{Limitaciones y futuras direcciones}

El presente estudio presenta algunas limitaciones que son importantes de destacar. Al momento de comprar la invarianza métrica del 
instrumento por sexo los tamaños muestrales utilizados se encuentran por debajo de lo recomendado (Beaujean, 2014); sin embargo, las diferencias en Chi-cuadrado no fueron tan dramáticas como para considerarlo un riesgo. En este sentido, al no encontrarse diferencias significativas en la clasificación de los grupos para hombres y mujeres se puede concluir que la probabilidad de ser clasificado en cualquiera de los grupos de estrategias de control de recursos es la misma para hombres y mujeres. Es recomendable para futuras investigaciones que este aspecto pueda ser corroborado.

Por otor lado, la medición utilizada para indicar el nivel de popularidad de los participantes del presente estudio fue de autoreporte, mientras que por lo general se recomienda utilizar mediciones sociométricas (Cillessen \& Marks, 2011). Lamentablemente, por restricciones de tiempo no se pudo realizar esta forma de medición, por lo que el error asociado esta variable podría ser mayor y esto daría cuenta en alguna medida la ausencia de resultados significativos en la comparación de grupos de estrategias de control de recursos. A pesar de esto las tendencias en los promedios se encuentran en la dirección de los resultados esperados, indicando que para futuras investigaciones es necesario utilizar medidas sociométricas con las variables de estatus para tener mas potencia estadística e identificar las posibles diferencias entre los grupos de estrategias de control de recursos.

En conclusión y a pesar de las limitaciones anteriormente mencionadas, el Inventario de Estrategias de Control de Recursos es un intrumento que permite conocer las conductas que instrumentalizan los escolares, en edad preadolescente, para obtener recursos sociales y materiales de sus pares. Las diferencias encontradas con los estudios de otras culturas frente a la chilena resaltan la necesidad de profundizar en investigaciones de la Teoría de Control de Recursos (Hawley, 1999) considerando la dimensión contextual y cultural, para que de este modo se pueda comprender de mejor manera las relaciones de pares en las escuelas latinoamericanas. 


\section{Referencias}

Beaujean, A. A. (2014). Latent Variable Modeling Using R: A Step by Step Guide. New York, NY: Routledge. https://doi. org/10.4324/9781315869780

Berger, C., \& Rodkin, P. C. (2012). Group Influences on Individual Aggression and Prosociality: Early Adolescents Who Change Peer Affiliations. Social Development, 21(2), 396-413. https:// doi.org/10.1111/j.1467-9507.2011.00628.x

Berger, C., Batanova, M., \& Cance, J. D. (2015). Aggressive and Prosocial? Examining Latent Profiles of Behavior, Social Status, Machiavellianism, and Empathy. Journal of Youth and Adolescence, 44(12), 2230-2244. https://doi.org/10.1007/ s10964-015-0298-9

Brislin, R. W. (1970). Back-Translation for Cross-Cultural Research. Journal of Cross-Cultural Psychology, 1(3), 185-216. https://doi. org/10.1177/135910457000100301

Cillessen, A. H. N. (2011). Toward a theory of Popularity. En A. H. N. Cilleseen, D. Schwartz, L. Mayeux (Eds.), Popularity in the peer system (pp. 273-299). New York, NY: Guilford Press.

Cillessen, A. H. N., Mayeux, L., Ha, T., de Bruyn, E. H., \& LaFontana, K. M. (2013). Aggressive effects of prioritizing popularity in early adolescence. Aggressive Behavior, 40(3), 204-213. https:// doi.org/10.1002/ab.21518

Crick, N. R., \& Dodge, K. A. (1994). A review and reformulation of the social-information processing mechanism in children's social adjustments. Psychological Bulletin, 115(1), 74-101. https://doi. org/10.1037/0033-2909.115.1.74

Findley-Van Nostrand, D., \& Ojanen, T. (2018). Forms of Prosocial Behaviors are Differentially Linked to Social Goals and Peer Status in Adolescents. The Journal of Genetic Psychology, 179(6), 329-342. https://doi.org/10.1080/00221325.2018.1518894

Findley, D., \& Ojanen, T. (2013). Adolescent resource control: Associations with physical and relational aggression, prosocial 
and withdrawn behaviors, and peer regard. International Journal of Behavioral Development, 37(6), 518-529. https://doi. org/10.1177/0165025413503420

Fuligni, A. J., Tseng, V. and Lam, M. (1999). Attitudes toward Family Obligations among American Adolescents with Asian, Latin American, and European Backgrounds. Child Development, 70(4), 1030-1044. https://doi.org/10.1111/1467-8624.00075

Hawley, P. H. (1999). The Ontogenesis of Social Dominance: A Strategy-Based Evolutionary Perspective. Developmental Review, 19(1), 97-132. https://doi.org/10.1006/drev.1998.0470

Hawley, P. H. (2003a). Strategies of control, aggression and morality in preschoolers: An evolutionary perspective. Journal of Experimental Child Psychology, 85, 213-235. https://doi. org/10.1016/S0022-0965(03)00073-0

Hawley, P. H. (2003b). Prosocial and coercive configurations of resource control in early adolescence: A case for the well-adapted Machiavellian. Merril-Palmer Quarterly, 49(3), 279-309. https:// doi.org/10.1353/mpq.2003.0013

Hawley, P. H. (2006). Resource Control Strategies Inventory - Revised. Lawrence, KS: University of Kansas.

Hawley, P. H. (2007). Social dominance in childhood and adolescence: Why social competence and aggression may go hand in hand. En P. H. Hawley, T. D. Little, \&, P. C. Rodkin (Eds.). Aggression and adaptation: The bright side to bad behavior (pp. 1-30). Mahwah, NJ: Lawrence Erlbaum Associates.

Hawley, P. H., Johnson, S. E., Mize, J. A., \& McNamara, K. A. (2007). Physical attractiveness in preschoolers: Relationships with power, status, aggression and social skills. Journal of School Psychology, 45(5), 499-521. https://doi.org/10.1016/j.jsp.2007.04.001

Hawley, P. H., Little, T. D., \&, Rodkin, P. C. (2007). Aggression and adaptation: The bright side to bad behavior. Mahwah: Lawrence Erlbaum Associates.

Hooper, D., Coughan, J., \& Mullen, M. R. (2008). Structural equation modelling: guidelines for determining model fit. The Electronic 
Journal of Business Research Methods, 6(1), 53-60. URL: http:// www.elbrm.com/

Olthof, T., Goossens, F. A., Vermande, M. M., Aleva, E. A., \& van der Meulen, M. (2011). Bullying as strategic behavior: Relations with desired and acquired dominance in the peer group. Journal of School Psychology, 49(3), 339-359. https://doi.org/10.1016/j. jsp.2011.03.003

Prinstein, M. J., \& Cillessen, A. H. (2003). Forms and Functions of Adolescent Peer Aggression Associated with High Levels of Peer Status. Merrill-Palmer Quarterly, 49(3), 310-342. https://doi. org/10.1353/mpq.2003.0015

R Core Team (2018). R: A language and environment for statistical computing. R Foundation for Statistical Computing, Vienna, Austria. URL http://www.R-project.org/

Rosseel, Y. (2012). lavaan: An R Package for Structural Equation Modeling. Journal of Statistical Software, 48(2), 1-36. https:// doi.org/10.18637/jss.v048.i02

Reijntjes, A., Vermande, M., Olthof, T., Goossens, F. A., Vink, G., Aleva, L., \& van der Meulen, M. (2017). Differences between resource control types revisited: A short term longitudinal study. Social Development, 27(1), 187-200. https://doi.org/10.1111/ sode. 12257

Recibido: $10 / 10 / 2019$

Revisado: $31 / 05 / 2020$

Aceptado: 06/06/2020 